\title{
Circulating LncRNA Serve as Fingerprint for Gestational Diabetes Mellitus Associated with Risk of Macrosomia
}

\author{
Jianfeng Lu Jinbao Wu Ziyu Zhao Jinmei Wang Zhong Chen \\ Department of Obstetrics and Gynecology, Wuxi Maternity and Child Health Hospital, Affiliated to \\ Nanjing Medical University, Wuxi, China
}

\section{Key Words}

Lncrna • Biomarker • Plasma • Macrosomia $・$ ROC

\begin{abstract}
Background/Aims: Fetal macrosomia and its associated complications are the most frequent and serious morbidities for infants associated with gestational diabetes mellitus (GDM). The aim of this study was to evaluate the IncRNAs involvement in GDM, especially for the prediction of risk for fetal overgrowth. Methods: The peripheral blood obtained from four group including healthy control (NC), healthy volunteers with pregnancy (NC-P), GDM patients with and without macrosomia were screened by IncRNA microarray and validated by quantitative real-time PCR (RT-qPCR) arranged in the training and a two-stage validation sets. The positive and negative prediction ability for candidate IncRNAs were analyzed by risk score analysis. Results: A multiple venny analysis was performed revealed five candidate IncRNA including XLOC_014172, RP11-230G5.2, PCBP1-AS1, LOC149086 and RP11-160H22.5 which was consistence with the following parameter: $i$, increased in GDM patients with macrosomia (GDM-M) comparing with patients without macrosomia; ii, increased in GDM-M comparing with NC-P group; iii, increased in GDM-M comparing with NC. Further validation found XLOC_014172 and RP11-230G5.2 was final consistence with these parameter in 150 samples each group. Further receiver operating characteristic curve $(R O C)$ analysis, with the combined two stably expressed IncRNAs indicated a high diagnostic ability an area under ROC curve value (AUC) of 0.955 and 0.962 in training set and validation set respectively. Conclusions: Circulating XLOC_014172 and RP11-230G5.2 may act as novel biomarkers in GDM patients as fingerprint for the risk of macrosomia outcome.

(C) 2018 The Author(s)

Published by S. Karger AG, Basel
\end{abstract}

\section{Introduction}

Gestational diabetes mellitus (GDM), defined as any degree of abnormal glucose tolerance with onset or first recognition during pregnancy, is one of the most common 
medical complications of pregnancy [1,2]. Babies born to mothers with GDM are typically at increased risk of excessive birth weight or macrosomia which defined as weighing more than $4 \mathrm{~kg}[3,4]$. Macrosomia is closely associated with higher rates of cesarean section and sequential use of vacuum and obstetric forceps for vaginal delivery for the mother, and clavicle fracture, intracranial hemorrhage and respiratory distress syndrome for the newborn $[5,6]$.

Recently, genetic studies have focused on non-coding RNAs. Over the past decade, many studies have reported that non-coding RNAs have important regulatory potentials, both transcriptionally and post transcriptionally [7-9]. LncRNAs, tentatively defined as noncoding RNAs more than 200 nucleotides, are usually divided into exonic lncRNAs, intronic lncRNAs, intergenic lncRNAs and overlapping lncRNAs in accordance with their location relative to the protein-coding transcripts $[10,11]$. LncRNAs are known to play important roles during cellular development and differentiation, and a large range of biological processes, such as modulation of tumor proliferation and invasiveness, and reprogramming of induced pluripotent stemcells $[12,13]$. However, to our knowledge to date, little study has been performed regarding the circulating lncRNAs for dynamic monitoring of GDM patients as well as the risk of macrosomia outcome.

In this study, we conducted our experiment based on the identified lncRNAs which was different expression in the GDM patients with or without macrosomia outcome. Five lncRNAs (XLOC_014172, RP11-230G5.2, PCBP1-AS1, LOC149086 and RP11-160H22.5) obtained as candidate fingerprints. We aimed to explore the potential lncRNA acting as fingerprint for the early and dynamic identification of macrosomia outcome in GDM patients.

\section{Materials and Methods}

\section{Samples and Screening phase}

This study was approved by an institutional review board, blood samples were collected after informed consent. Women with GDM, diagnosed by 75g oral glucose tolerance test (OGTT) during their 24th to 28th week of gestation at the Obstetrics and Gynecology Department in Wuxi Maternity and Child Health Hospital in 2011-2016 were enrolled in this study. According to the IADPSG (The International Association of Diabetes and Pregnancy Study Groups) and ADA (American Diabetes Association) recommendation. GDM was diagnosed if any one glucose level was equal to or greater than fasting $5.1 \mathrm{mmol} / \mathrm{L}$, one hour post load $10.0 \mathrm{mmol} / \mathrm{L}$ or two hours post load $8.5 \mathrm{mmol} / \mathrm{L}$. Pregnant women with GDM were treated with a strict diet control to maintain the fasting plasma glucose level at less than $5.3 \mathrm{mmol} / \mathrm{L}$ and $2 \mathrm{~h}$ postprandial level at less than $6.7 \mathrm{mmol} / \mathrm{L}$. Insulin treatment was administrated when dietary control could not achieve this goal. The babies of GDM group were diagnosed as macrosomia when their birth-weight was equal to or exceeded 4000 $\mathrm{g}[14,15]$. Controls were from healthy volunteers without any health problems during their health checkups at the at the Obstetrics and Gynecology Department in Wuxi Maternity and Child Health Hospital. The research protocol was approved by the Institutional Ethics Committee of the Wuxi Maternity and Child Health Hospital. Written informed consent was obtained from every participant. The peripheral blood samples of patients were collected in the early pregnancy during the antenatal examination period. Blood samples were collected in a separate vacuum cube, followed by centrifugation at 3, $000 \mathrm{rpm}$ for $10 \mathrm{~min}$. All samples were stored at $-80^{\circ} \mathrm{C}$ until further analysis. The relevant clinical data of all patients were available. The detailed information of patients and healthy controls was summarized in Table1.

Table 1. Clinical characteristics of selected variables in patients with GDM and controls

\begin{tabular}{lcccccccccc}
\hline \multirow{2}{*}{ Characteristics } & \multicolumn{2}{c}{ NC(n=150) } & \multicolumn{2}{c}{ NC-P(n=150) } & GDM (n=150) & GDM-M(n=150) & \multirow{2}{*}{ P(GDM-M /NC) } & P(GDM-M/GDM) \\
& Mean & Mean & Mean & SEM & Mean & SEM & Mean & SEM & & \\
\hline Age (years) & 32 & 3 & 33 & 2 & 31 & 4 & 32 & 3 & 0.12 & 0.13 \\
Pregestational BMI (kg/m2) & 18.552 & 2.112 & 19.949 & 2.228 & 21.11 & 3.189 & 20.309 & 2.156 & 0.19 & 0.22 \\
Fasting plasma glucosea(mmol/L) & 4.532 & 0.449 & 4.703 & 0.608 & 5.002 & 1.118 & 5.018 & 1.202 & 0.0004 & 0.38 \\
OGTT 0h (mmol/L) & 4.33 & 0.956 & 4.55 & 0.599 & 5.181 & 1.967 & 5.036 & 1.301 & 0.0003 & 0.41 \\
OGTT 1h (mmol/L) & 7.661 & 1.881 & 7.709 & 1.256 & 10.894 & 1.552 & 10.094 & 1.625 & 0.0003 & 0.28 \\
OGTT 2h (mmol/L) & 6.991 & 0.332 & 6.818 & 0.806 & 7.989 & 1.334 & 8.591 & 2.399 & 0.0002 & 0.17 \\
Glycated hemoglpbin protein $(\%)$ & 4.55 & 0.512 & 4.73 & 0.608 & 6.11 & 0.982 & 5.72 & 0.698 & 0.0003 & 0.34 \\
Cholesterol (mmol/L) & 5.521 & 0.771 & 5.972 & 0.797 & 5.887 & 0.579 & 5.981 & 0.905 & 0.79 & 0.42 \\
Triglyceride (mmol/L) & 2.442 & 1.302 & 2.705 & 1.045 & 2.501 & 1.889 & 2.795 & 1.382 & 0.22 & 0.17 \\
HDL cholesterol (mmol/L) & 2.221 & 0.567 & 2.309 & 0.689 & 2.321 & 0.489 & 2.273 & 0.599 & 0.25 & 0.29 \\
LDL cholesterol (mmol/L) & 3.118 & 0.597 & 3.263 & 0.752 & 0.699 & 0.677 & 3.209 & 0.716 & 0.18 & 0.31 \\
\end{tabular}




\section{Cellular Physiology Cell Physiol Biochem 2018;48:1012-1018 \begin{tabular}{c|c} 
DOI: 10.1159/000491969 & $\begin{array}{l}\text { O 2018 The Author(s). Published by S. Karger AG, Basel } \\
\text { www.karger.com/cpb }\end{array}$
\end{tabular}}

Lu et al.: LncRNA as Biomarker for GDM-M

The screening phase was divided into training set and validation set. 20 samples each group were enrolled while the validation set contained two cohorts. Groups were named as healthy controls (NC), pregnant volunteer without DM (NC-P), GDM patients without macrosomia (GDM) and GDM patients with macrosomia (GDM-M). The detailed workflow was presented in Fig. 1. The sample number in training set was 20 participants in each group while 130 participants in each group pf validation set.

\section{Training set}

All candidates were tested in an independent cohort of 20 plasma samples obtained from

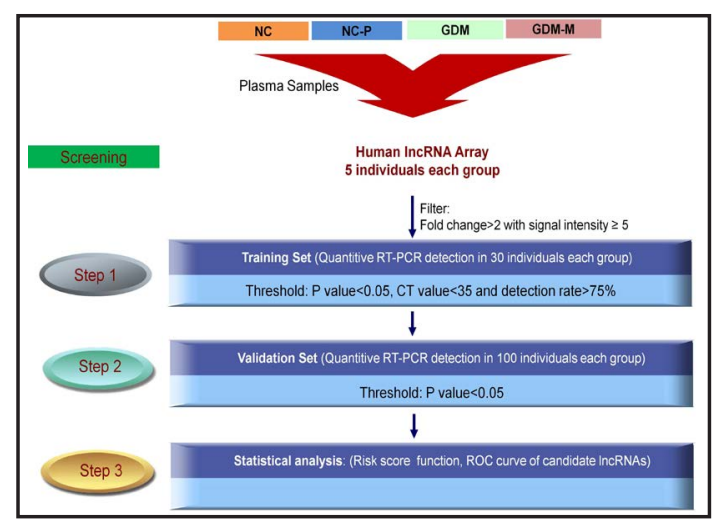

Fig. 1. Workflow of multiple analysis for candidate lncRNA screening. patients. The expression levels of these candidates were analyzed in all samples, and comparative $2^{-\Delta \Delta C t}$ method algorithms were used to analyze the difference between different groups.

\section{Validation set}

A case-control study was designed to validate the obvious difference of relative expression levels of the selected potential biomarkers candidates in another independent cohort including 130 samples each group.

\section{Quantitative real-time PCR ( $q R T-P C R)$}

Total RNAs were obtained from plasmic samples using TRIzol reagent as described by the manufacturer (Invitrogen Life Technologies Co, Carlsbad, CA, USA). For mRNA detection, total RNAs (500ng) were reverse transcribed using the reverse transcription kit (Takara, Tokyo, Japan). The quality of RNA samples was assessed by a UV spectrophotometer (Bio-Rad, Hercules, CA, USA), and the 260/280nm absorbance ratio of samples were limited to 1.8-2.0. QRT-PCR was performed by using ABI Prism 7900HT (Applied Biosystems, CA, USA).

\section{Risk score analysis}

Risk score analysis was performed to evaluate the associations between the concentrations of the plasma lncRNA expression levels. The upper 95\% reference interval of each IncRNA value in controls or non-metastasis group was set as the threshold to code the expression level of the corresponding lncRNA for each sample as 0 and 1 in the training set. A risk score function (RSF) to predict NSCLC group was defined according to a linear combination of the expression level for each lncRNA. For example, the RSF for sample i using information from three lncRNAs was: $r s f i=\sum 3 j-1 W j . s i j$. In the above equation, sij is the risk score for lncRNA $\mathrm{j}$ on sample $\mathrm{i}$, and $\mathrm{Wj}$ is the weight of the risk score of lncRNA $\mathrm{j}$. The risk score of three lncRNAs was calculated using the weight by the regression coefficient that was derived from the univariate logistic regression analysis of each lncRNAs. Samples were ranked according to their RSF and then divided into a high-risk group, representing the GDM-M group, and a low-risk group, representing the predicted control individuals. Frequency tables and ROC curves were then used to evaluate the diagnostic effects of the profiling and to find the appropriate cutoff point, and to validate the procedure and cutoffs in the next validation sample set.

\section{Statistical analysis}

Data were presented as mean (S.E.M.) If there is no special case. The Student's t-test and MannWhitney unpaired test analysis of variance were used to evaluate statistical differences between patients and controls. Analysis of area under the ROC curve (AUC) was used to estimate the effectiveness of lncRNAs for prediction. Statistical analysis was performed using softwares of STATA 10.0 and GraphPad prism. In all cases, $P<0.05$ was considered to be significant. All $P$ values were two-sided. 


\section{Results}

\section{Subject characteristics}

The clinical characteristics of the GDM patients and other groups were presented in Table 1. GDM patients presented with higher plasma glucose both during fasting and during OGTT, and higher HbA1C and triglycerides than control pregnant women $(\mathrm{P}<0.001)$. HDL cholesterol was significantly lower in GDM subjects $(\mathrm{P}<0.05)$. No difference were obtained in GDM-M group comparing with the GDM group (Table 1).

\section{The circulating IncRNA expression landscape in GDM} patients

Human LncRNA Array v3.0 was used to detect the IncRNA derived from plasma of 12 sample including three peripheral blood samples from healthy volunteer, three from pregnant volunteer without DM (NC-P), and three from GDM patients without macrosomia and three from GDM patients with macrosomia. Aberrant expression of lncRNA underwent hierarchical clustering using a heat map, resulting in a profile of the differential expression of lncRNA in the different groups (Fig. 2A). Among these, we identified 284 increased lncRNAs in pregnant volunteer without DM comparing with healthy control without pregnancy; 197 increased lncRNAs in GDM patients without macrosomia comparing with pregnant volunteer without DM and 147 increased lncRNAs in GDM patients with macrosomia comparing with GDM patients without macrosomia according to a fold-change cut-off of $4 / 0.25$. To further screened the potential fingerprints for GDM as predicting the macrosomia out in gravidae, we next using the following parameter for screening: i, increased in GDM patients with macrosomia (GDM-M) comparing with patients without macrosomia; ii, increased in GDM-M comparing with NC-P group; iii, increased in GDM-M comparing with NC. Finally, we obtained five candidate lncRNAs which was consistence with all the parameters mentioned above including XLOC_014172, RP11-230G5.2, PCBP1-AS1, LOC149086 and RP11-160H22.5 (Fig. 2B).

Fig. 2. LncRNA microarray and expression of increased IncRNAs A: Hierarchical cluster analysis of lncRNA microarray. B: Expression of two significant lncRNAs profiling in plasma samples from healthy volunteer, pregnant volunteer without DM, GDM patients without macrosomia and GDM patients with macrosomia outcome. Each group including 150 samples. Data was presented as mean \pm SEM. Data was analyzed with student $t$ test. * indicated $\mathrm{p}<0.05$; ** indicated $\mathrm{p}<0.01$.

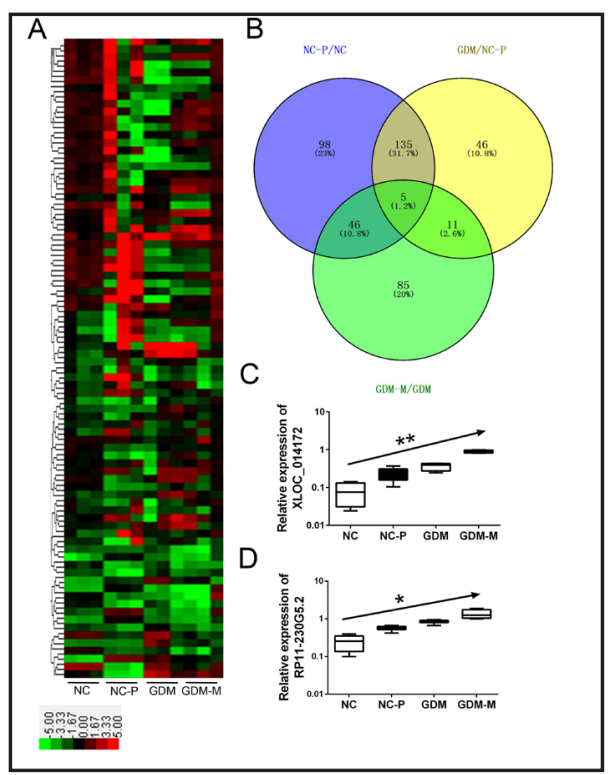


Fig. 3. Relative expression of lncRNAs with no significant difference. Relative expression of the rest lncRNAs profiling in plasma samples from healthy volunteer, pregnant volunteer without DM, GDM patients without macrosomia and GDM patients with macrosomia outcome. Each group including 150 samples. Data was presented as mean \pm SEM. Data was analyzed with student $t$ test.
Fig. 4. The ROC analysis based on risk score. B: ROC curve analysis was conducted for discrimination between GDM patients without macrosomia and GDM patients with macrosomia outcome in training set. Each group including 20 samples. C: GDM patients without macrosomia and GDM patients with macrosomia outcome in validation set. Each group including 130 samples. The detailed area under ROC curve value was presented below.


Validation of candidate lncRNAs in large sample size

We next examined their expression levels through a larger individual samples (150 samples each group). As shown in Fig. 2C, we found that only XLOC_014172 and RP11230G5.2 were consisted with the training set while PCBP1-AS1, LOC149086 and RP11$160 \mathrm{H} 22.5$ indicated no significant difference (Fig. 3).

To validate the accuracy and specificity of the two lncRNAs as a GDM with macrosomia outcome potential signature, the risk score formula was applied to assess the diagnostic value of the two IncRNAs profiling system. Firstly, the risk score of each plasma sample in the training set was calculated (20 samples random selection each group), as the basis of their risk scores and a set cut off, plasma samples were then divided into a high-risk group, representing the possible GDM-M group, and a low-risk group, representing the GDM group. At the optimal cutoff value (Value $=9.889$ ) with the value of sensitivity + specificity considered to be maximal, the positive predictive value (PPV) and negative predictive value (NPV) was $70 \%$ and $80 \%$ in the training set, respectively. Similarly, when the same cutoff value was applied to calculate the risk score of samples in the larger validation sets, the PPV and NPV were $85 \%, 86 \%$, respectively (Table2).

ROC curve analysis was used to evaluate the predicting diagnosis value of lncRNAs for GDM-M. Areas under the ROC curves (AUC) of the two lncRNAs signature in training set were 0.900 and 0.885 , respectively while the combination of the two factors possessed a moderate ability for discrimination between GDM-M and GDM with an area under the ROC curve of 0.955 (Fig. 4A). Additional, in the validation set, AUC of the two lncRNAs signature in training set were 0.904 and 0.849 , respectively while the combination of the two factors possessed a moderate ability for discrimination between GDM-M and GDM with an area under the ROC curve of 0.962 (Fig. 4B) 


\section{Discussion}

Gestational diabetes mellitus is a complex disease induced by a combination of genetic factors and environmental exposure, with genetic components being the major determinant of disease [16]. To identify specific lncRNA signature and its biological significance in the pathobiology of GDM, we performed IncRNA profiling in plasma samples from healthy volunteer, pregnant volunteer without DM, GDM patients without macrosomia and GDM patients with macrosomia outcome. In our study, we performed a case-control study through the high throughput IncRNA microarray, and discovered two novel IncRNA, XLOC_014172 and RP11-230G5.2, acting as the biomarker for predicting the outcome of macrosomia in GDM patients. The risk score analysis including a multistage validation was employed to evaluate the association between GDM-M group and the lncRNAs expression levels.

It has been widely reported that disease-associated lncRNAs are detectable in blood, sputum, urine, and other biological fluids of patients $[17,18]$. Besides, miRNA such as miR508-3p has been identified as signature in gestational diabetes mellitus and was associated with risk of macrosomia [19]. For IncRNA, researcher has reported the deregulated MALAT1 expression in patients with gestational diabetes mellitus; however, no detailed analysis was applied for predicting the GDM or the outcome of macrosomia in GDM patients [20]. Therefore, IncRNA might be investigated as potential biomarkers for the dynamic monitoring of GDM. The function of IncRNA has been focused by other project, for example, Shi Z, et al, reported a microarray expression profile analysis of IncRNA in umbilical cord plasma samples of GDM patients, they revealed a landscape of lncRNA in patients suffering from GDM-induced macrosomia [21]; however, no mechanism study or diagnosis analysis was performed. Moreover, alteration in expression and methylation of lncRNA igf $2 / \mathrm{h} 19$ in placenta and umbilical cord blood were also associated with macrosomia exposed to intrauterine hyperglycemia [22].

From a clinical point of view, the IncRNA biomarker selected in our study for the monitoring of GDM-induced macrosomia might be useful for indicating the clinical typing of GDM which is a great help to decide the way of the treatment. Furthermore, for as a single diagnostic method, the biomarker in our study presented a high sensitivity and specificity, nevertheless, given that the express fluctuations of plasma lncRNA analysis with a panel of multiple diagnosis methods might be a solution to the problem of GDM detection.

In conclusion, we have identified the unique lncRNA biomarker for early screening of GDM-induced macrosomia in GDM patients, which may serve as a novel non-invasive approach for diagnosis and dynamic monitoring of GDM-induced macrosomia.

\section{Disclosure Statement}

The authors declare to have no competing interests.

\section{References}

-1 Reulen RC, Bright CJ, Winter DL, Fidler MM, Wong K, Guha J, Kelly JS, Frobisher C, Edgar AB, Skinner R, Wallace WHB, Hawkins MM: Pregnancy and Labor Complications in Female Survivors of Childhood Cancer: The British Childhood Cancer Survivor Study. J Natl Cancer Inst 2017;109.

-2 Garnaes KK, Morkved S, Salvesen O, Moholdt T: Exercise Training and Weight Gain in Obese Pregnant Women: A Randomized Controlled Trial (ETIP Trial). PLoS Med 2016;13:e1002079.

3 Vaarasmaki M: Is it worth treating gestational diabetes: if so, when and how? Diabetologia 2016;59:13911395.

4 Ravnsborg T, Andersen LL, Trabjerg ND, Rasmussen LM, Jensen DM, Overgaard M: First-trimester multimarker prediction of gestational diabetes mellitus using targeted mass spectrometry. Diabetologia 2016;59:970-979. 


\section{Cellular Physiology Cell Physiol Biochem 2018;48:1012-1018 \begin{tabular}{l|l} 
DOI: 10.1159/000491969 & O 2018 The Author(s). Published by S. Karger AG, Basel \\
www.karger.com/cpb
\end{tabular}}

Lu et al.: LncRNA as Biomarker for GDM-M

5 Bao W, Tobias DK, Bowers K, Chavarro J, Vaag A, Grunnet LG, Strom M, Mills J, Liu A, Kiely M, Zhang C: Physical activity and sedentary behaviors associated with risk of progression from gestational diabetes mellitus to type 2 diabetes mellitus: a prospective cohort study. JAMA Intern Med 2014;174:1047-1055.

6 Wei Q, Sun Z, Yang Y, Yu H, Ding H, Wang S: Effect of a CGMS and SMBG on Maternal and Neonatal Outcomes in Gestational Diabetes Mellitus: a Randomized Controlled Trial. Sci Rep 2016;6:19920.

7 Meng J, Li P, Zhang Q Yang Z, Fu S: A four-long non-coding RNA signature in predicting breast cancer survival. J Exp Clin Cancer Res 2014;33:84.

-8 Tong YS, Wang XW, Zhou XL, Liu ZH, Yang TX, Shi WH, Xie HW, Lv J, Wu QQ, Cao XF: Identification of the long non-coding RNA POU3F3 in plasma as a novel biomarker for diagnosis of esophageal squamous cell carcinoma. Mol Cancer 2015;14:3.

-9 Boon RA, Jae N, Holdt L, Dimmeler S: Long Noncoding RNAs: From Clinical Genetics to Therapeutic Targets? J Am Coll Cardiol 2016;67:1214-1226.

10 Tang J, Zhuo H, Zhang X, Jiang R, Ji J, Deng L, Qian X, Zhang F, Sun B: A novel biomarker Linc00974 interacting with KRT19 promotes proliferation and metastasis in hepatocellular carcinoma. Cell Death Dis 2014;5:e1549.

11 Li J, Wang X, Tang J, Jiang R, Zhang W, Ji J, Sun B: HULC and Linc00152 Act as Novel Biomarkers in Predicting Diagnosis of Hepatocellular Carcinoma. Cell Physiol Biochem 2015;37:687-696.

12 Yan Y, Zhang B, Liu N, Qi C, Xiao Y, Tian X, Li T, Liu B: Circulating Long Noncoding RNA UCA1 as a Novel Biomarker of Acute Myocardial Infarction. Biomed Res Int 2016;2016:8079372.

13 Tang J, Jiang R, Deng L, Zhang X, Wang K, Sun B: Circulation long non-coding RNAs act as biomarkers for predicting tumorigenesis and metastasis in hepatocellular carcinoma. Oncotarget 2015;6:4505-4515.

14 Agha-Jaffar R, Oliver N, Johnston D, Robinson S: Gestational diabetes mellitus: does an effective prevention strategy exist? Nat Rev Endocrinol 2016;12:533-546.

15 Kew S, Ye C, Sermer M, Connelly PW, Hanley AJ, Zinman B, Retnakaran R: Postpartum metabolic function in women delivering a macrosomic infant in the absence of gestational diabetes mellitus. Diabetes Care 2011;34:2608-2613.

-16 Poulsen P, Levin K, Petersen I, Christensen K, Beck-Nielsen H, Vaag A: Heritability of insulin secretion, peripheral and hepatic insulin action, and intracellular glucose partitioning in young and old Danish twins. Diabetes 2005;54:275-283.

17 Salem ESB, Fan GC: Pathological Effects of Exosomes in Mediating Diabetic Cardiomyopathy. Adv Exp Med Biol 2017;998:113-138.

18 Crea F, Clermont PL, Parolia A, Wang Y, Helgason CD: The non-coding transcriptome as a dynamic regulator of cancer metastasis. Cancer Metastasis Rev 2014;33:1-16.

19 Li J, Song L, Zhou L, Wu J, Sheng C, Chen H, Liu Y, Gao S, Huang W: A MicroRNA Signature in Gestational Diabetes Mellitus Associated with Risk of Macrosomia. Cell Physiol Biochem 2015;37:243-252.

20 Zhang Y, Wu H, Wang F, Ye M, Zhu H, Bu S: Long non-coding RNA MALAT1 expression in patients with gestational diabetes mellitus. Int J Gynaecol Obstet 2018;140:164-169.

-21 Shi Z, Zhao C, Long W, Ding H, Shen R: Microarray Expression Profile Analysis of Long Non-Coding RNAs in Umbilical Cord Plasma Reveals their Potential Role in Gestational Diabetes-Induced Macrosomia. Cell Physiol Biochem 2015;36:542-554.

-22 Su R, Wang C, Feng H, Lin L, Liu X, Wei Y, Yang H: Alteration in Expression and Methylation of IGF2/ H19 in Placenta and Umbilical Cord Blood Are Associated with Macrosomia Exposed to Intrauterine Hyperglycemia. PLoS One 2016;11:e0148399. 